\title{
Breakdown of Synchronization in Chaotic Oscillators and Noisy Oscillators
}

\author{
Ryo Imabayashi, Yoko Uwate and Yoshifumi Nishio \\ Department of Electrical and Electronic Engineering, Tokushima University, \\ 2-1 Minami-Josanjima, Tokushima 770-8506, JAPAN \\ Telephone: +81-88-656-7470, Fax: +81-88-656-7471 \\ Email:\{bayashi, uwate, nishio\}@ee.tokushima-u.ac.jp
}

\begin{abstract}
In this study, the breakdown of synchronization observed from four coupled chaotic oscillators is investigated. In order to understand the phenomenon, the model of coupled modified van der Pol oscillators with noise is considered. The comparison of the coupled chaotic oscillators with the coupled modified van der Pol oscillators with noise gives us some interesting results.
\end{abstract}

\section{INTRODUCTION}

Synchronization is one of typical nonlinear phenomena observed in the field of natural science. There have been many investigations on mutual synchronization of oscillators. Breakdown of synchronization is a kind of cooperative phenomenon for dissipated assembly oscillators and is important to clarify its mechanism for better understanding of higher-dimensional complicated phenomena [1]-[6]. In the case of coupled chaotic oscillators, chaotic fluctuations of their waveforms are supposed to play a role to break the synchronization and the breakdown sometimes causes chaotic wandering phenomenon.

On the other hand, there is certainly noise in actual physical systems. In these years, investigations on systems including noise attract many researchers' attentions. We are interested in the difference between chaotic fluctuation and noise, because sometimes chaos exhibits better performance than random noise in information processing tasks [7].

In this study, we investigate the breakdown of synchronization observed from four coupled chaotic oscillators. In order to understand the phenomenon, we consider the model of coupled modified van der Pol oscillators with noise. The comparison of the coupled chaotic oscillators with the coupled modified van der Pol oscillators with noise gives us some interesting results.

\section{Circuit Model}

\section{A. Chaotic Oscillators}

Figure 1 shows the circuit model. In this circuit, four identical chaotic oscillators are coupled by one resistor. By changing the variables and the parameters,

$$
\begin{aligned}
& I_{k}=\sqrt{\frac{C}{L_{1}}} E x_{k}, \quad i_{k}=\sqrt{\frac{C}{L_{1}}} E y_{k}, \quad v_{k}=E z_{k}, \\
& t=\sqrt{L_{1} C} \tau, \quad \alpha=\frac{L_{1}}{L_{2}}, \quad \beta=r \sqrt{\frac{C}{L_{1}}} \\
& \gamma=R \sqrt{\frac{C}{L_{1}}}, \quad \delta=r_{d} \sqrt{\frac{C}{L_{1}}}
\end{aligned}
$$

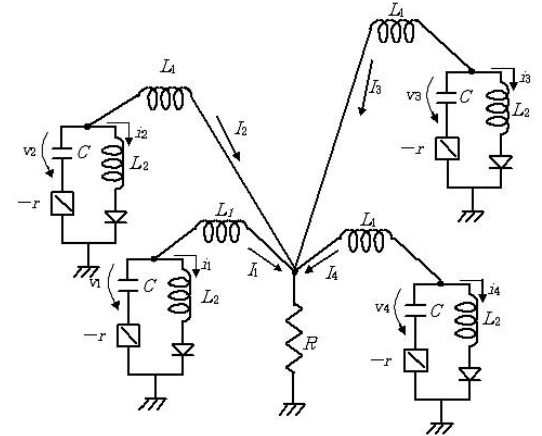

Fig. 1. Coupled chaotic oscillators.

the normalized circuit equations of the circuit are described as

$$
\left\{\begin{array}{l}
\frac{d x_{k}}{d \tau}=\beta\left(x_{k}+y_{k}\right)-z_{k}-\gamma \sum_{j=1}^{4} x_{j} \\
\frac{d y_{k}}{d \tau}=\alpha\left\{\beta\left(x_{k}+y_{k}\right)-z_{k}-f\left(y_{k}\right)\right\} \\
\frac{d z_{k}}{d \tau}=x_{k}+y_{k} \quad(k=1,2,3,4)
\end{array}\right.
$$

where

$$
f\left(y_{k}\right)=0.5\left(\delta y_{k}+1-\left|\delta y_{k}-1\right|\right) .
$$

Figure 2 shows an example of the observed four-phase quasi-synchronization of chaos. In the figures the phase differences of $x_{2}, x_{3}$ and $x_{4}$ with respect to $x_{1}$ are almost $90^{\circ}$, $180^{\circ}$ and $270^{\circ}$, respectively.

Because of symmetry of the coupling structure, six different combinations of phase states coexist;

$$
\begin{aligned}
& S_{1}:\left(0^{\circ}, 90^{\circ}, 180^{\circ}, 270^{\circ}\right), \\
& S_{2}:\left(0^{\circ}, 90^{\circ}, 270^{\circ}, 180^{\circ}\right), \\
& S_{3}:\left(0^{\circ}, 180^{\circ}, 90^{\circ}, 270^{\circ}\right), \\
& S_{4}:\left(0^{\circ}, 180^{\circ}, 270^{\circ}, 90^{\circ}\right), \\
& S_{5}:\left(0^{\circ}, 270^{\circ}, 90^{\circ}, 180^{\circ}\right), \\
& S_{6}:\left(0^{\circ}, 270^{\circ}, 180^{\circ}, 90^{\circ}\right) .
\end{aligned}
$$

As the coupling resistance $R(\gamma)$ decreases, we can observe that the synchronization states become unstable and that the self-switching phenomenon of the states occurs [6]. The breakdown of the synchronization means the start of the self-switching, hence it is important to investigate how the synchronization breaks down. 


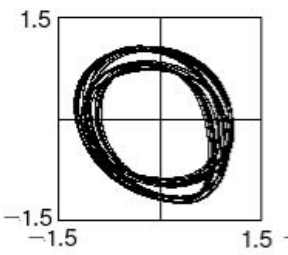

(a)

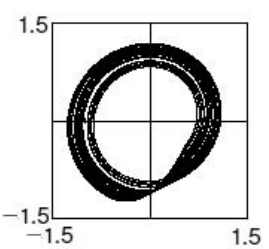

(d)

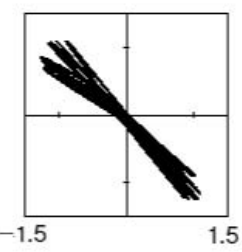

(b)

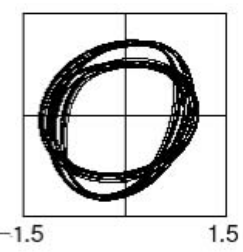

(c)

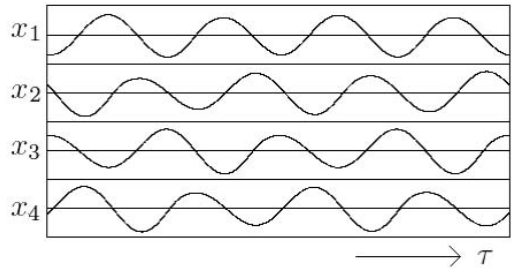

(e)

Fig. 2. Four-phase quasi-synchronization of chaos (computer calculated result). $\alpha=7.0, \beta=0.10, \gamma=0.10$ and $\delta=100.0$. (a) $x_{1}$ vs. $x_{2}$. (b) $x_{1}$ vs. $x_{3}$. (c) $x_{1}$ vs. $x_{4}$. (d) $x_{1}$ vs. $z_{1}$. (e) Time waveforms.

\section{B. Modified van der Pol Oscillators}

Next, we consider four coupled van der Pol oscillators. In order to obtain the waveforms similar to those of the

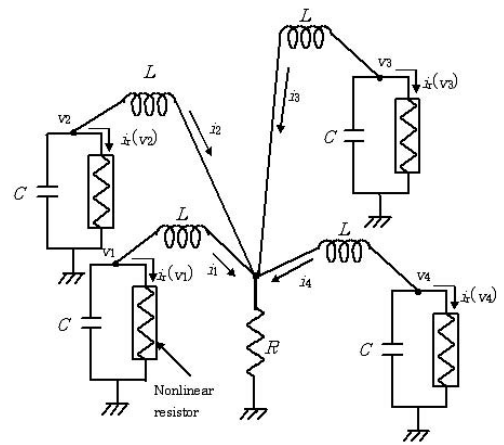

Fig. 3. Coupled van der Pol oscillators.

chaotic oscillator, we modify the van der Pol oscillator with the nonlinear resistor whose $v-i$ characteristics are described by the following asymmetric function

$$
i_{r}\left(v_{k}\right)=-g_{1} v_{k}+g_{2} v_{k}^{2}+g_{3} v_{k}^{3} \quad\left(g_{1}, g_{2}, g_{3}>0\right) .
$$

By changing the variables and the parameters,

$$
\begin{gathered}
v_{k}=\sqrt{\frac{g_{1}}{g_{3}}} x_{k}, \quad i_{k}=\sqrt{\frac{C g_{1}}{L g_{3}}} y_{k}, \quad t=\sqrt{L C} \tau, \\
\nu=\frac{g_{2}}{\sqrt{g_{1} g_{3}}}, \quad \gamma=R \sqrt{\frac{C}{L}}, \quad \varepsilon=g_{1} \sqrt{\frac{L}{C}},
\end{gathered}
$$

the normalized circuit equations are given as

$$
\left\{\begin{array}{l}
\frac{d x_{k}}{d \tau}=\xi\left\{-y_{k}+\varepsilon\left(x_{k}-\nu x_{k}^{2}-x_{k}^{3}\right)\right\} \\
\frac{d y_{k}}{d \tau}=x_{k}-\gamma \sum_{j=1}^{4} y_{j} \\
(k=1,2,3,4)
\end{array}\right.
$$

where $\xi$ is the parameter added to tune the period of the waveform.
Figure 4 shows an example of the observed four-phase synchronization of the modified van der Pol oscillators.

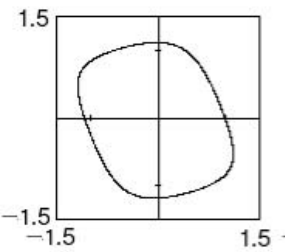

(a)

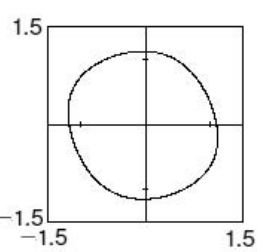

(d)

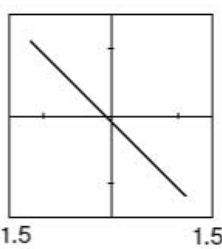

(b)

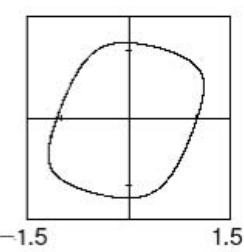

(c)

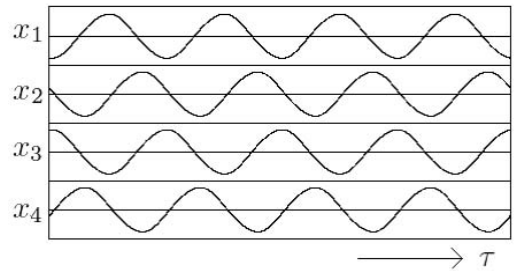

(e)

Fig. 4. Four-phase synchronization of modified van der Pol oscillators (computer calculated result). $\varepsilon=0.50, \gamma=0.60, \xi=1.07$ and $\nu=0.1035$. (a) $x_{1}$ vs. $x_{2}$. (b) $x_{1}$ vs. $x_{3}$. (c) $x_{1}$ vs. $x_{4}$. (d) $x_{1}$ vs. $y_{1}$. (e) Time waveforms.

In this study, we consider the case that the noise is added to the modified van der Pol oscillator in order to simulate the breakdown of the synchronization in the coupled chaotic oscillators caused by chaotic fluctuations of their waveforms.

When we add the noise to the voltage amplitude of the modified van der Pol oscillator, the circuit equation of the coupled oscillators are described as

$$
\left\{\begin{aligned}
& \frac{d x_{k}}{d \tau}= \xi\left[-y_{k}+\varepsilon\left\{(1+\sigma(\tau)) x_{k}\right.\right. \\
&\left.\left.-\nu\left((1+\sigma(\tau)) x_{k}\right)^{2}-\left((1+\sigma(\tau)) x_{k}\right)^{3}\right\}\right] \\
& \frac{d y_{k}}{d \tau}=(1+\sigma(\tau)) x_{k}-\gamma \sum_{j=1}^{4} y_{j} \\
&(k=1,2,3,4)
\end{aligned}\right.
$$

where $\sigma(\tau)$ is the added noise.

While when we add the noise to the voltage period of the modified van der Pol oscillator, the circuit equation of the coupled oscillators are described as

$$
\left\{\begin{array}{l}
\frac{d x_{k}}{d \tau}=(1+\sigma(\tau)) \xi\left\{-y_{k}+\varepsilon\left(x_{k}-\nu x_{k}^{2}-x_{k}^{3}\right)\right\} \\
\frac{d y_{k}}{d \tau}=x_{k}-\gamma \sum_{j=1}^{4} y_{j} \\
(k=1,2,3,4) .
\end{array}\right.
$$

In this study we concentrate on the case that the added noise is the additive white Gaussian noise (AWGN) with the average 0 and the variance $\sigma^{2}$.

\section{BREAKDOWN OF SYNCHRONIZATION}

When the coupling parameter $\gamma$ is relatively large, both the coupled chaotic oscillators and the modified van der Pol oscillators with noise exhibit four phase synchronizations. 
While for relatively smaller $\gamma$, the synchronizations break down and we observe the switchings of phase states. This means that a critical value of the coupling parameter exists which divides the parameter space into the synchronization region and the self-switching region. We define this critical coupling parameter as $\gamma_{c}$ and investigate how $\gamma_{c}$ changes when the strength of chaos or noise increases.

First, we define the breakdown of synchronization as at least one switching during 50,000 periods. Next, in order to compare the coupled chaotic oscillators with the modified van der Pol oscillators, we set the parameters as follows. We investigate the critical coupling parameter $\gamma_{c}$ for the coupled chaotic oscillators when the chaotic oscillator exhibits the first period-doubling bifurcation $(\beta=0.0425)$. This value $\gamma_{c}=1.189$ is set as the standard value. We tune the parameter of the modified van der Pol oscillators without noise in order to the synchronization breaks down at this standard value of $\gamma_{c}$. By this setting of the parameter, the chaotic oscillators without chaotic fluctuation and the modified van der Pol oscillators without noise are equalized in the sense of the stability of the synchronization.

Figures 5 and 6 show one-parameter bifurcation diagram of the chaotic oscillator and the critical coupling parameter $\gamma_{c}$, respectively. In Fig. 6, the region over the curve corresponds to the synchronization region and the region under the curve corresponds to the self-switching region. We can observe that $\gamma_{c}$ increases as $\beta$ increases. This means that the synchronization becomes easier to be broken down for larger $\beta$, namely larger chaotic fluctuation.

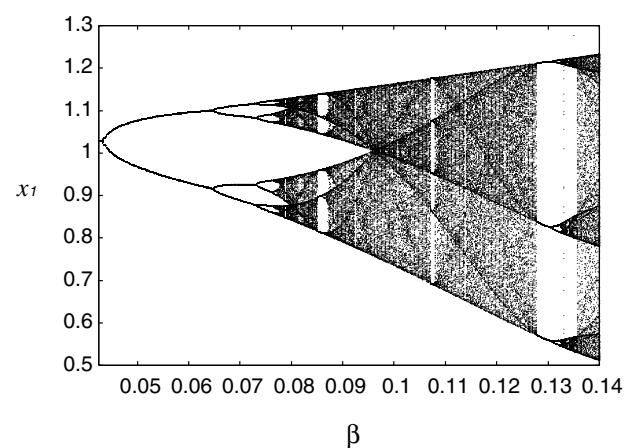

Fig. 5. One-parameter bifurcation diagram of chaotic oscillator $(\alpha=7.0$, $\gamma=0.0$ and $\delta=100.0$ ).

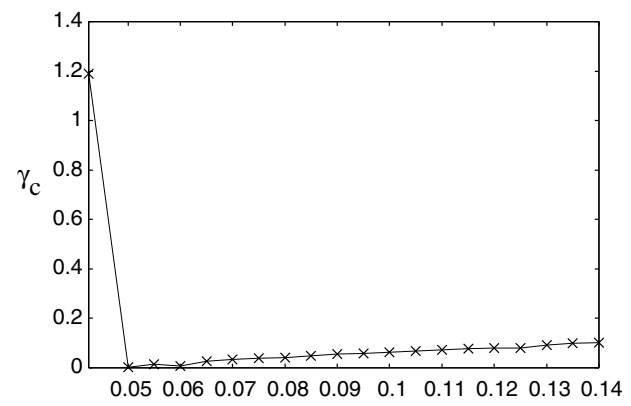

$\beta$

Fig. 6. Breakdown of synchronization of coupled chaotic oscillators $(\alpha=7.0$ and $\delta=100.0$ ).
Figure 7 shows the critical coupling parameter $\gamma_{c}$ of the coupled modified van der Pol oscillators when the noise is added to their voltage amplitude. The horizontal axis shows the standard deviation of the added noise $\sigma$. For $\sigma=0.20$, $\gamma_{c}$ takes the minimum value. When $\sigma$ becomes larger beyond $0.20, \gamma_{c}$ increases.

Figure 8 shows the critical coupling parameter $\gamma_{c}$ of the coupled modified van der Pol oscillators when the noise is added to their voltage period. Similar to the previous case, for $\gamma_{c}$ takes the minimum value $\sigma=0.20$ and $\gamma_{c}$ increases for $\sigma>0.20$

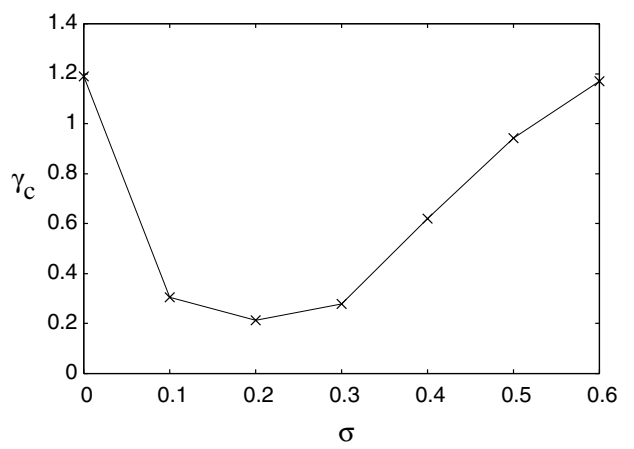

Fig. 7. Breakdown of synchronization of modified van der Pol oscillators with noisy amplitude $(\varepsilon=0.5, \xi=1.07$ and $\nu=0.1035)$.

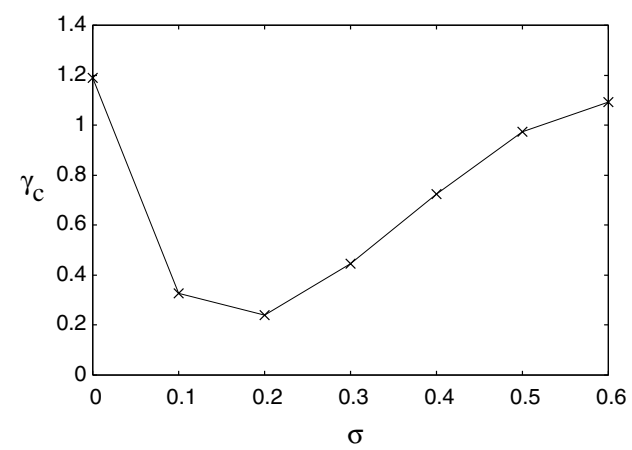

Fig. 8. Breakdown of synchronization of modified van der Pol oscillators with noisy period $(\varepsilon=0.5, \xi=1.07$ and $\nu=0.1035)$.

We noticed that the values of $\gamma_{c}$ for the coupled modified van der Pol oscillators are much larger than those for the coupled chaotic oscillators. Although we have to investigate more precisely before concluding, chaotic systems may be synchronized more stably than simple periodic oscillators with noise.

\section{Distribution of Amplitude And Period}

In order to evaluate the effect of the noise added to the coupled modified van der Pol oscillators, we compare the statistical characteristics of amplitudes and periods of the chaotic oscillator with the noisy modified van der Pol oscillators.

The distributions of the voltage amplitude are shown in Figs. 9-11. The distributions of the voltage period are shown in Figs. 12-14. 


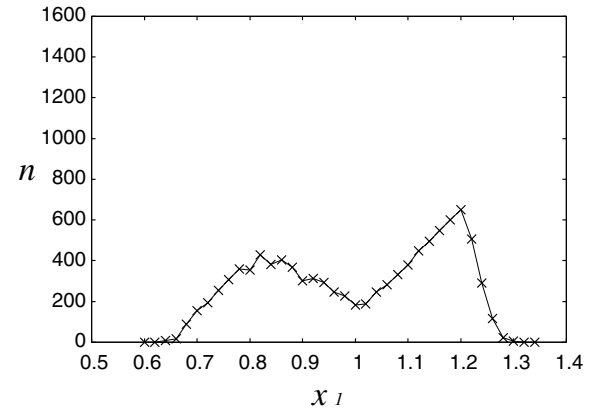

Fig. 9. Distribution of voltage amplitude of chaotic oscillators $(\alpha=7.0$, $\beta=0.1, \gamma=0.065$ and $\delta=100.0$ ).

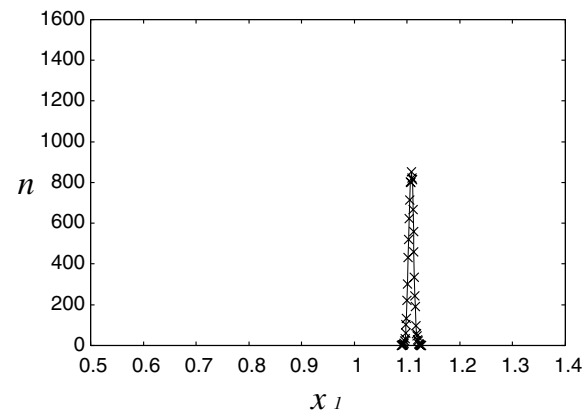

Fig. 10. Distribution of voltage amplitude of modified van der Pol oscillators with noisy amplitude $(\gamma=0.942, \varepsilon=0.5, \xi=1.07, \nu=0.1035$ and $\sigma=0.5$ ).

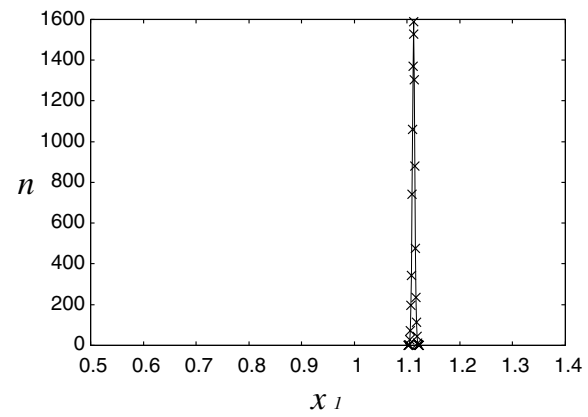

Fig. 11. Distribution of voltage amplitude of modified van der Pol oscillators with noisy period $(\gamma=0.973, \varepsilon=0.5, \xi=1.07, \nu=0.1035$ and $\sigma=0.5)$.

The distributions show the difference between the chaotic oscillator and the modified van der Pol oscillator with noise. Namely, the chaotic oscillator shows a kind of two-band characteristics, while the modified van der Pol oscillator shows the normal distribution. We consider that this may be the main reason of the difference on the breakdown of the synchronization.

\section{CONCLUSION}

In this study, the breakdown of synchronization observed from four coupled chaotic oscillators has been investigated. In order to understand the phenomenon, the model of coupled modified van der Pol oscillators with noise was considered. The simulation results suggested that chaotic systems might be synchronized more stably than simple periodic oscillators with noise. We also compared the statistical characteristics of the amplitudes and the periods of the chaotic oscillator with the noisy modified van der Pol oscillators and considered that their difference might be the reason of the difference on the breakdown of the synchronization.

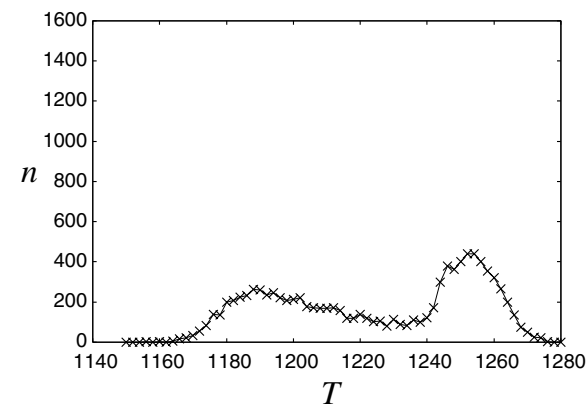

Fig. 12. Distribution of voltage period of chaotic oscillators $(\alpha=7.0$, $\beta=0.1, \gamma=0.065$ and $\delta=100.0$ ).

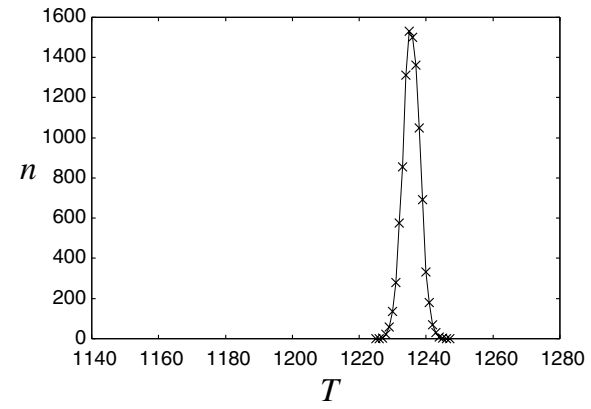

Fig. 13. Distribution of voltage period of modified van der Pol oscillators with noisy amplitude $(\gamma=0.942, \varepsilon=0.5, \xi=1.07, \nu=0.1035$ and $\sigma=0.5)$.

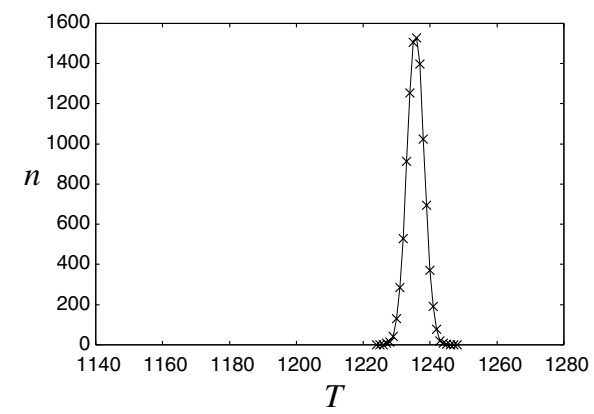

Fig. 14. Distribution of voltage period of modified van der Pol oscillators with noisy period $(\gamma=0.973, \varepsilon=0.5, \xi=1.07, \nu=0.1035$ and $\sigma=0.5$ ).

\section{ACKOWLEDGMENTS}

This work was partly supported by Yazaki Memorial Foundation for Science and Technology.

\section{REFERENCES}

[1] N. Platt, E.A. Spiegel and C. Tresser, "On-Off Intermittency: A Mechanism for Bursting,” Phys. Rev. Lett., vol. 70, no. 3, pp. 279-282, 1993.

[2] E. Ott and J.C. Sommerer, "Blowout Bifurcations: the Occurrence of Riddled Basins and On-Off Intermittency," Phys. Lett., vol. A188, no. 3, pp. 39-47, 1994.

[3] P. Ashwin, J. Buescu and I. Stewart, "Bubbling of Attractors and Synchronization of Chaotic Oscillators," Phys. Rev. Lett., vol. A193, no. 3, pp. 126-139, 1994.

[4] T. Kapitaniak and L.O. Chua, "Locally-Intermingled Basins of Attraction in Coupled Chua's Circuits," Int. J. Bifurcation and Chaos, vol. 6, no. 2, pp. 357-366, 1996.

[5] M. Wada, Y. Nishio and A. Ushida, "Analysis of Bifurcation Phenomena on Two Chaotic Circuits Coupled by an Inductor," IEICE Trans. Fundamentals, vol. E80-A, no. 5, pp. 869-875, 1997.

[6] Y. Nishio and A. Ushida, "Chaotic Wandering and its Analysis in Simple Coupled Chaotic Circuits," IEICE Trans. on Fundamentals, vol. E85-A, no. 1, pp. 248-255, 2002.

[7] Y. Hayakawa and Y. Sawada, "Effects of the Chaotic Noise on the Performance of a Neural Network Model for Optimization Problems," Phys. Rev. E, vol. 51, no. 4, pp. 2693-2696, 1995. 